Homology, Homotopy and Applications, vol.13(1), 2011, pp.297-318

\title{
CYCLIC STRUCTURES IN ALGEBRAIC (CO)HOMOLOGY THEORIES
}

\author{
NIELS KOWALZIG AND ULRICH KRÄHMER
}

(communicated by Jean-Louis Loday)

\begin{abstract}
This note discusses the cyclic cohomology of a left Hopf algebroid $\left(\times_{A}\right.$-Hopf algebra $)$ with coefficients in a right module-left comodule, defined using a straightforward generalisation of the original operators given by Connes and Moscovici for Hopf algebras. Lie-Rinehart homology is a special case of this theory. A generalisation of cyclic duality that makes sense for arbitrary para-cyclic objects yields a dual homology theory. The twisted cyclic homology of an associative algebra provides an example of this dual theory that uses coefficients that are not necessarily stable anti Yetter-Drinfel'd modules.
\end{abstract}

\section{Introduction}

\subsection{Topic}

A left Hopf algebroid ( $\times_{A}$-Hopf algebra) $U$ is, roughly speaking, a Hopf algebra whose ground ring is not a field $k$ but a possibly noncommutative $k$-algebra $A$ [B2, Sch2]. The concept provides, in particular, a natural framework for unifying and extending classical constructions in homological algebra. Group, Lie algebra, Hochschild, and Poisson homology are all special cases of Hopf algebroid homology

$$
H_{\bullet}(U, M):=\operatorname{Tor}_{\bullet}^{U}(M, A), \quad M \in U^{\mathrm{op}} \text {-Mod, }
$$

since the rings $U$ over which these theories can be expressed as derived functors are all left Hopf algebroids. This allows one, for example, to study cup and cap products as well as the phenomenon of Poincaré duality in a uniform way $[\mathbf{K o K r}]$.

Similarly, we describe here how the additional structure of a left $U$-comodule on $M$ induces a para-cyclic structure (cf. Section 2.7) on the canonical chain complex $C_{\text {. }}(U, M)$ that computes $H_{\bullet}(U, M)$ assuming $U$ is flat over $A$. This defines, in particular, an analogue of the Connes-Rinehart-Tsygan differential

$$
B: H_{\bullet}(U, M) \rightarrow H_{\bullet+1}(U, M) .
$$

Assuming a suitable compatibility between the $U$-action and the $U$-coaction (namely that $M$ is a stable anti Yetter-Drinfel'd module), the para-cyclic $k$-module $C$. $(U, M)$

Received December 12, 2010, revised April 28, 2011; published on June 6, 2011.

2000 Mathematics Subject Classification: 16T05, 16E40; 16T15, 19D55, 58B34.

Key words and phrases: cyclic homology, Hopf algebroid, twisted cyclic homology, Lie-Rinehart algebra.

Article available at http://intlpress.com/HHA/v13/n1/a11 and doi:10.4310/HHA.2011.v13.n1.a11

Copyright (C) 2011, International Press. Permission to copy for private use granted. 
is, in fact, cyclic and hence turned by $B$ into a mixed complex. However, we will also discuss concrete examples which demonstrate the necessity to go beyond this setting.

\subsection{Background}

The operator $B$ has been defined by Rinehart on the Hochschild homology of a commutative $k$-algebra $A$ (with $M=A$ and $U=A^{\mathrm{e}}=A \otimes_{k} A^{\mathrm{op}}$ ) in order to define the de Rham cohomology of an arbitrary affine scheme over $k[\mathbf{R i}]$. Connes and Tsygan independently rediscovered it around 1980 as a central ingredient in their definition of cyclic homology which extends Rinehart's theory to noncommutative algebras [C, FTs].

Connes and Moscovici, and Crainic initiated the study of the case of a Hopf algebra $U$ over $A=k$ with one-dimensional coefficients $M[\mathbf{C M 2}, \mathbf{C r}]$. The class of admissible coefficient modules $M$ was subsequently enlarged to stable anti Yetter-Drinfel'd modules [HKhRS1, HKhRS2], and Kaygun finally obtained the construction for Hopf algebras with arbitrary modules-comodules as coefficients [Ka1, Ka2].

Noncommutative base rings appeared for the first time in the particular example of the "extended" Hopf algebra governing the transversal geometry of foliations [CM1]. The general theory has then been further developed in [BŞ1, BŞ2, HasR, KhR, Ko, KoP, Ma].

\subsection{Results}

Our first aim here is to give explicit formulas for the most straightforward generalisation of the original operators defined by Connes and Moscovici in [CM1] towards Hopf algebroids and completely general coefficients. We copy the result here; see the main text for the details and, in particular, for the notation used:

Theorem 1.1. Let $U$ be a left Hopf algebroid over a k-algebra $A$ and $M$ be a right $U$-module and left $U$-comodule with compatible induced left $A$-module structures. Then $C \cdot(U, M):=U^{\otimes_{A}} \cdot \otimes_{A} M$ carries a canonical para-cocyclic $k$-module structure with codegeneracies and cofaces

$$
\begin{aligned}
& \delta_{i}\left(z \otimes_{A} m\right)= \begin{cases}1 \otimes_{A} u^{1} \otimes_{A} \cdots \otimes_{A} u^{n} \otimes_{A} m & \text { if } i=0, \\
u^{1} \otimes_{A} \cdots \otimes_{A} \Delta\left(u^{i}\right) \otimes_{A} \cdots \otimes_{A} u^{n} \otimes_{A} m & \text { if } 1 \leqslant i \leqslant n, \\
u^{1} \otimes_{A} \cdots \otimes_{A} u^{n} \otimes_{A} m_{(-1)} \otimes_{A} m_{(0)} & \text { if } i=n+1,\end{cases} \\
& \delta_{j}(m)= \begin{cases}1 \otimes_{A} m & \text { if } j=0, \\
m_{(-1)} \otimes_{A} m_{(0)} & \text { if } j=1,\end{cases} \\
& \sigma_{i}\left(z \otimes_{A} m\right)=u^{1} \otimes_{A} \cdots \otimes_{A} \epsilon\left(u^{i+1}\right) \otimes_{A} \cdots \otimes_{A} u^{n} \otimes_{A} m \quad 0 \leqslant i \leqslant n-1,
\end{aligned}
$$

where $z=u^{1} \otimes_{A} \cdots \otimes_{A} u^{n}$, and with cocyclic operator

$$
\tau_{n}\left(z \otimes_{A} m\right)=u_{-(1)}^{1} u^{2} \otimes_{A} \cdots \otimes_{A} u_{-(n-1)}^{1} u^{n} \otimes_{A} u_{-(n)}^{1} m_{(-1)} \otimes_{A} m_{(0)} u_{+}^{1} .
$$

The proof closely follows the literature cited above, which contains similar constructions of para-cyclic and para-cocyclic modules assigned to Hopf algebroids (compare especially [BŞ2, Prop. 2.19]). However, there seems no reference for the exact setting we consider here. In particular, Kaygun's pivotal observation that Theorem 1.1 is true for arbitrary modules-comodules over $k$-bialgebras has to our knowledge not 
been extended to noncommutative base rings so far. Last but not least, the above also answers the question of how the Hopf-cyclic (co)homologies in $[\mathbf{K o}, \mathbf{K o P}]$ can be extended to general coefficients.

Also, it has been pointed out by several authors that the standard operation of cyclic duality, which canonically identifies cyclic and cocyclic objects, does not lift to para-(co)cyclic objects; see, e.g., [BŞ1]. However, in Section 4 we show that a different choice of anti-autoequivalence of the cyclic category leads to a form of cyclic duality that does lift. This allows us to construct in full generality a cyclic dual $\left(C .(U, M), d_{\bullet}, s_{\bullet}, t_{\bullet}\right)$ from the para-cocyclic module from Theorem 1.1. We provide an isomorphism of this with the para-cyclic module $M \otimes_{A^{\mathrm{op}}}\left(\sim_{\triangleleft}\right)^{\otimes_{A^{\mathrm{op}}}} \cdot$ whose structure maps are given by

$$
\begin{aligned}
& d_{i}\left(m \otimes_{A^{\mathrm{op}}} x\right)= \begin{cases}m \otimes_{A^{\mathrm{op}}} u^{1} \otimes_{A^{\mathrm{op}}} \cdots \otimes_{A^{\mathrm{op}}} \epsilon\left(u^{n}\right) u^{n-1} & \text { if } i=0, \\
m \otimes_{A^{\mathrm{op}}} \cdots \otimes_{A^{\mathrm{op}}} u^{n-i} u^{n-i+1} \otimes_{A^{\mathrm{op}}} \cdots & \text { if } 1 \leqslant i \leqslant n-1, \\
m u^{1} \otimes_{A^{\mathrm{op}}} u^{2} \otimes_{A^{\mathrm{op}}} \cdots \otimes_{A^{\mathrm{op}}} u^{n} & \text { if } i=n,\end{cases} \\
& s_{i}\left(m \otimes_{A^{\mathrm{op}}} x\right)= \begin{cases}m \otimes_{A^{\mathrm{op}}} u^{1} \otimes_{A^{\mathrm{op}}} \cdots \otimes_{A^{\mathrm{op}}} u^{n} \otimes_{A^{\mathrm{op}} 1} 1 & \text { if } i=0, \\
m \otimes_{A^{\mathrm{op}}} \cdots \otimes_{A^{\mathrm{op}}} u^{n-i} \otimes_{A^{\mathrm{op}}} 1 \otimes_{A^{\mathrm{op}}} u^{n-i+1} \otimes_{A^{\mathrm{op}}} \cdots & \text { if } 1 \leqslant i \leqslant n-1, \\
m \otimes_{A^{\mathrm{op}}} 1 \otimes_{A^{\mathrm{op}}} u^{1} \otimes_{A^{\mathrm{op}}} \cdots \otimes_{A^{\mathrm{op}}} u^{n} & \text { if } i=n,\end{cases} \\
& t_{n}\left(m \otimes_{A^{\mathrm{op}}} x\right)=m_{(0)} u_{+}^{1} \otimes_{A^{\mathrm{oP}}} u_{+}^{2} \otimes_{A^{\mathrm{op}}} \cdots \otimes_{A^{\mathrm{op}}} u_{+}^{n} \otimes_{A^{\mathrm{op}}} u_{-}^{n} \cdots u_{-}^{1} m_{(-1)},
\end{aligned}
$$

where we abbreviate $x:=u^{1} \otimes_{A^{\text {op }}} \cdots \otimes_{A^{\text {op }}} u^{n}$.

It is precisely this variation of Hopf-cyclic theory that has the ordinary Hopf algebroid homology as the underlying simplicial homology, and, in particular, the one which reduces to the original cyclic homology of an associative algebra when one applies it to the Hopf algebroid $U=A^{\mathrm{e}}$. Now the freedom to consider arbitrary coefficients becomes crucial since it allows one, for example, to incorporate the twisted cyclic homology of Kustermans, Murphy and Tuset [KuMuTu]. This paper has been the first one to generalise the Connes-Rinehart-Tsygan operator $B$ on the Hochschild homology of an associative algebra to coefficients in $(A, A)$-bimodules other than $A$ itself, namely those where one of the two actions of $A$ on itself is twisted by an algebra automorphism $\sigma$. In the last section we will explain how this example fits into the above Hopf-cyclic homology theory, and, in particular, we will observe that these coefficients are not SaYD modules. As an important classical case of the Hopfcyclic cohomology theory from Theorem 1.1, we will also consider the example of Lie-Rinehart homology.

\section{Preliminaries}

\subsection{Some conventions}

Throughout this note, "ring" means "unital and associative ring", and we fix a commutative ring $k$. All other algebras, modules etc., will each have an underlying structure of a $k$-module. We also fix a $k$-algebra $A$, i.e., a ring with a ring homomorphism $\eta_{A}: k \rightarrow Z(A)$ to its centre. We denote by $A$-Mod the category of left $A$-modules, by $A^{\text {op }}$ the opposite and by $A^{\mathrm{e}}:=A \otimes_{k} A^{\text {op }}$ the enveloping algebra of $A$. An $A$-ring is a monoid in the monoidal category $\left(A^{\mathrm{e}}\right.$-Mod, $\left.\otimes_{A}, A\right)$ of $A^{\mathrm{e}}$-modules (i.e., $(A, A)$-bimodules with symmetric action of $k$ ), fulfilling associativity and unitality. 
Likewise, an $A$-coring is a comonoid in $\left(A^{\mathrm{e}}\right.$-Mod, $\left.\otimes_{A}, A\right)$, fulfilling coassociativity and counitality.

Our main object is an $A^{\mathrm{e}}$-ring $U$ (a monoid in $\left(A^{\mathrm{e}} \otimes_{k} A^{\mathrm{e}}\right)$-Mod). Explicitly, such an $A^{\mathrm{e}}$-ring is given by a $k$-algebra homomorphism $\eta=\eta_{U}: A^{\mathrm{e}} \rightarrow U$ whose restrictions

$$
s:=\eta\left(-\otimes_{k} 1\right): A \rightarrow U \text { and } t:=\eta\left(1 \otimes_{k}-\right): A^{\mathrm{op}} \rightarrow U
$$

will be called the source and target map, respectively. Left and right multiplication in $U$ give rise to an $\left(A^{\mathrm{e}}, A^{\mathrm{e}}\right)$-bimodule structure on $U$, that is, four commuting actions of $A$ that we denote by

$$
a \triangleright u \triangleleft b:=s(a) t(b) u, \quad a \triangleright u \triangleleft b:=u s(b) t(a), \quad a, b \in A, u \in U .
$$

If not stated otherwise, we view $U$ as an $(A, A)$-bimodule using the actions $\triangleright, \triangleleft$. In particular, we define the tensor product $U \otimes_{A} U$ with respect to this bimodule structure. Using the actions $\bullet, \triangleleft$, then permits one to define the Takeuchi product

$$
U \times_{A} U:=\left\{\sum_{i} u_{i} \otimes_{A} v_{i} \in U \otimes_{A} U \mid \sum_{i} a \bullet u_{i} \otimes_{A} v_{i}=\sum_{i} u \otimes_{A} v_{i} \triangleleft a, \forall a \in A\right\} .
$$

This is an $A^{\mathrm{e}}$-ring via factorwise multiplication. Similarly, $\operatorname{End}_{k}(A)$ is an $A^{\mathrm{e}}$-ring with ring structure given by composition and $(A, A)$-bimodule structure $(a \varphi b)(c):=$ $\varphi(b c a), \varphi \in \operatorname{End}_{k}(A), a, b, c \in A$.

\subsection{Bialgebroids $[\mathrm{T}]$}

Bialgebroids are a generalisation of bialgebras. An important subtlety is that the algebra and coalgebra structure are defined in different monoidal categories.

Definition 2.1. Let $A$ be a $k$-algebra. A left bialgebroid over $A$ (or $A$-bialgebroid or $\times_{A}$-bialgebra) is an $A^{\mathrm{e}}$-ring $U$ together with two homomorphisms of $A^{\mathrm{e}}$-rings

$$
\Delta: U \rightarrow U \times{ }_{A} U, \quad \hat{\epsilon}: U \rightarrow \operatorname{End}_{k}(A),
$$

which turn $U$ into an $A$-coring with coproduct $\Delta$ (viewed as a map $U \rightarrow U \otimes_{A} U$ ) and counit $\epsilon: U \rightarrow A, u \mapsto(\hat{\epsilon}(u))(1)$.

Note that this means, for example, that $\epsilon$ satisfies for all $u, v \in U$

$$
\epsilon(u v)=\epsilon(u \triangleleft \epsilon(v))=\epsilon(\epsilon(v) \bullet u) .
$$

Analogously one defines right bialgebroids where the roles of $\triangleright, \triangleleft$ and $\bullet, \triangleleft$ are exchanged. We shall not write out the details, but rather refer to $[\mathbf{K S z}, \mathbf{B 2}]$.

\subsection{Left Hopf algebroids [Sch2]}

Left Hopf algebroids have been introduced by Schauenburg under the name $\times_{A^{-}}$Hopf algebras and generalise Hopf algebras towards left bialgebroids. For a left bialgebroid $U$ over $A$, one defines the (Hopf-)Galois map

$$
\beta: U_{A^{\text {op }}} U \triangleleft \rightarrow U_{\triangleleft} \otimes_{A} \triangleright U, \quad u \otimes_{A^{\text {op }}} v \mapsto u_{(1)} \otimes_{A} u_{(2)} v,
$$

where

$$
\text { - } U \otimes_{A^{\mathrm{op}}} U \triangleleft=U \otimes_{k} U / \operatorname{span}\left\{a \triangleright u \otimes_{k} v-u \otimes_{k} v \triangleleft a \mid u, v \in U, a \in A\right\} .
$$

Definition 2.2 ([Sch2]). A left $A$-bialgebroid $U$ is called a left Hopf algebroid (or $\times_{A}$-Hopf algebra) if $\beta$ is a bijection. 
In a similar manner, one defines right Hopf algebroids (cf. [BSz, Prop. 4.2]). Following [Sch2], we adopt a Sweedler-type notation

$$
u_{+} \otimes_{A^{\mathrm{op}}} u_{-}:=\beta^{-1}\left(u \otimes_{A} 1\right)
$$

for the so-called translation map $\beta^{-1}\left(-\otimes_{A} 1\right): U \rightarrow \circlearrowleft \otimes_{A^{\text {op }}} U_{\triangleleft}$. Useful for our subsequent calculations, one has for all $u, v \in U, a \in A$ [Sch2, Prop. 3.7]:

$$
\begin{aligned}
u_{+}(1) \otimes_{A} u_{+(2)} u_{-} & =u \otimes_{A} 1 \in U \triangleleft \otimes_{A} \triangleright U, \\
u_{(1)+} \otimes_{A^{\mathrm{op}}} u_{(1)-} u_{(2)} & =u \otimes_{A^{\mathrm{op}}} 1 \in \checkmark \otimes_{A^{\mathrm{op}}} U \triangleleft, \\
u_{+} \otimes_{A^{\mathrm{op}}} u_{-} & \in U \times_{A^{\mathrm{op}}} U, \\
u_{+}(1) \otimes_{A} u_{+(2)} \otimes_{A^{\mathrm{op}}} u_{-} & =u_{(1)} \otimes_{A} u_{(2)+} \otimes_{A^{\mathrm{op}}} u_{(2)-}, \\
u_{+} \otimes_{A^{\mathrm{op}}} u_{-(1)} \otimes_{A} u_{-(2)} & =u_{++} \otimes_{A^{\mathrm{op}}} u_{-} \otimes_{A} u_{+-}, \\
(u v)_{+} \otimes_{A^{\mathrm{op}}}(u v)_{-} & =u_{+} v_{+} \otimes_{A^{\mathrm{op}}} v_{-} u_{-}, \\
u_{+} u_{-} & =s(\epsilon(u)), \\
u_{+} t\left(\epsilon\left(u_{-}\right)\right) & =u, \\
(s(a) t(b))_{+} \otimes_{A^{\mathrm{op}}}(s(a) t(b))_{-} & =s(a) \otimes_{A^{\mathrm{op}}} s(b),
\end{aligned}
$$

where in (6) we mean the Takeuchi product

$U \times_{A^{\mathrm{op}}} U:=\left\{\sum_{i} u_{i} \otimes_{A^{\mathrm{op}}} v_{i} \in \boldsymbol{\diamond} U \otimes_{A^{\mathrm{op}}} U \triangleleft \mid \sum_{i} u_{i} \triangleleft a \otimes_{A^{\mathrm{op}}} v_{i}=\sum_{i} u_{i} \otimes_{A^{\mathrm{op}}} a \triangleleft v_{i}\right\}$,

which is an algebra by factorwise multiplication, but with opposite multiplication on the second factor. Note that in (8) the tensor product over $A^{\text {op }}$ links the first and third tensor component. By (4) and (6) one can write

$$
\beta^{-1}\left(u \otimes_{A} v\right)=u_{+} \otimes_{A^{\text {op }}} u_{-} v,
$$

which is easily checked to be well-defined over $A$ with (9) and (11).

Remark 2.3. Observe that there is no notion of antipode for a left Hopf algebroid. Böhm and Szlachányi have introduced the concept of a (full or two-sided) Hopf algebroid [B2], which is, roughly speaking, an algebra equipped with a left and a right bialgebroid structure over anti-isomorphic base algebras $A$ and $B$, together with an antipode mapping from the left bialgebroid to the right. However, it is proved in $[\mathbf{B S z}$, Prop. 4.2] that a full Hopf algebroid with invertible antipode can be equivalently described as an algebra with both a left and a right Hopf algebroid structure subject to compatibility conditions, which motivates one to speak of left Hopf algebroids rather than $\times_{A}$-Hopf algebras.

\section{4. $U$-modules}

Let $U$ be a left bialgebroid with structure maps as before. Left and right $U$-modules are defined as modules over the ring $U$, with respective actions denoted by juxtaposition or, at times, by a dot for the sake of clarity. We denote the respective categories by $U$-Mod and $U^{\text {op }}$-Mod; while $U$-Mod is a monoidal category, $U^{\mathrm{op}}$-Mod is, in general, not [Sch1]. One has a forgetful functor $U$-Mod $\rightarrow A^{\mathrm{e}}$-Mod using which we consider every left $U$-module $N$ also as an $(A, A)$-bimodule with actions

$$
a n b:=a \triangleright n \triangleleft b:=s(a) t(b) n, \quad a, b \in A, n \in N .
$$

Similarly, every right $U$-module $M$ is also an $(A, A)$-bimodule via 


$$
a m b:=a \bullet m \triangleleft b:=m s(b) t(a), \quad a, b \in A, m \in M,
$$

and in both cases we usually prefer to express these actions just by juxtaposition if no ambiguity is to be expected.

\section{5. $U$-comodules}

Similarly as for coalgebras, one may define comodules over bialgebroids, but the underlying $A$-module structures need some extra attention. For the following definition see, e.g., [Sch1, B1, BrzWi].

Definition 2.4. A left $U$-comodule for a left bialgebroid $U$ over $A$ is a left comodule of the underlying $A$-coring $(U, \Delta, \epsilon)$, i.e., a left $A$-module $M$ with action $L_{A}:(a, m) \mapsto$ am and a left $A$-module map

$$
\Delta_{M}: M \rightarrow U_{\triangleleft} \otimes_{A} M, \quad m \mapsto m_{(-1)} \otimes_{A} m_{(0)}
$$

satisfying the usual coassociativity and counitality axioms

$$
(\Delta \otimes \mathrm{id}) \circ \Delta_{M}=\left(\mathrm{id} \otimes \Delta_{M}\right) \circ \Delta_{M} \quad \text { and } \quad L_{A} \circ(\epsilon \otimes \mathrm{id}) \circ \Delta_{M}=\mathrm{id} .
$$

We denote the category of left $U$-comodules by $U$-Comod.

Analogously, one defines right $U$-comodules and comodules for right bialgebroids. On any left $U$-comodule one can additionally define a right $A$-action

$$
m a:=\epsilon\left(m_{(-1)} s(a)\right) m_{(0)} .
$$

This is the unique action that turns $M$ into an $A^{\mathrm{e}}$-module in such a way that the coaction is an $A^{\mathrm{e}}$-module morphism

$$
\Delta_{M}: M \rightarrow U \times{ }_{A} M,
$$

where $U \times{ }_{A} M$ is the Takeuchi product

$$
U \times_{A} M:=\left\{\sum_{i} u_{i} \otimes_{A} m_{i} \in U \otimes_{A} M \mid \sum_{i} u_{i} t(a) \otimes_{A} m_{i}=\sum_{i} u_{i} \otimes_{A} m_{i} a, \forall a \in A\right\} .
$$

As a result, $\Delta_{M}$ satisfies the identities

$$
\begin{aligned}
\Delta_{M}(a m b) & =s(a) m_{(-1)} s(b) \otimes_{A} m_{(0)}, \\
m_{(-1)} \otimes_{A} m_{(0)} a & =m_{(-1)} t(a) \otimes_{A} m_{(0)} .
\end{aligned}
$$

This is compatible with (14) since one has $\epsilon(u s(a))=\epsilon(u t(a))$ for all $u \in U, a \in A$.

One can then prove (see [B2, Thm. 3.18] and [Sch1, Prop. 5.6]) that $U$-Comod has a monoidal structure such that the forgetful functor $U$-Comod $\rightarrow A^{\mathrm{e}}$-Mod is monoidal: for any two comodules $M, M^{\prime} \in U$-Comod, their tensor product $M \otimes_{A} M^{\prime}$ is a left $U$-comodule by means of the coaction

$$
\begin{aligned}
\Delta_{M \otimes_{A} M^{\prime}}: M \otimes_{A} M^{\prime} & \rightarrow U \otimes_{A}\left(M \otimes_{A} M^{\prime}\right), \\
m \otimes_{A} m^{\prime} & \mapsto m_{(-1)} m_{(-1)}^{\prime} \otimes_{A} m_{(0)} \otimes_{A} m_{(0)}^{\prime} .
\end{aligned}
$$

The map $\Delta_{M \otimes_{A} M^{\prime}}$ is easily checked to be well-defined.

Remark 2.5. If $\sigma \in U$ is a grouplike element in a (left) bialgebroid, then the formulas

$$
{ }_{A} \Delta(a):=t(a) \sigma \quad \text { and } \quad \Delta_{A}(a):=s(a) \sigma, \quad a \in A,
$$

define right and left $U$-comodule structures on $A$, which we shall refer to as induced by $\sigma$. In particular, the base algebra $A$ carries for any bialgebroid both canonical right 
and left coactions induced by $\sigma=1$, contrasting the fact that $A$ carries in general only a canonical left $U$-module structure induced by $\epsilon$, but no right one.

Remark 2.6. A special feature for bialgebroids $U$ over commutative base algebras $A$ with $s=t$ is that every left $A$-module $M$ can be made, for example, into a left $U$ comodule by means of the trivial coaction $m \mapsto 1 \otimes_{A} m$ (it follows from (15) that this is not possible in general).

\subsection{Stable anti Yetter-Drinfel'd modules}

The following definition is the left bialgebroid right module and left comodule version of the corresponding notion in [BŞ2]. For Hopf algebras, the concept goes back to [HKhRS1].

Definition 2.7. Let $U$ be a left Hopf algebroid with structure maps as before, and let $M$ simultaneously be a left $U$-comodule with coaction denoted as above and a right $U$-module with action denoted by $(m, u) \mapsto m u$ for $u \in U, m \in M$. We call $M$ an anti Yetter-Drinfel'd ( $a Y D$ ) module provided the following holds:

1. The $A^{\mathrm{e}}$-module structure on $M$ originating from its nature as $U$-comodule coincides with the $A^{\mathrm{e}}$-module structure induced by the right $U$-action on $M$, i.e., for all $a, b \in A$ and $m \in M$ we have

$$
a m b=a \bullet m \triangleleft b,
$$

where the right $A$-module structure on the left-hand side is given by (14).

2. For $u \in U$ and $m \in M$, one has

$$
\Delta_{M}(m u)=u_{-} m_{(-1)} u_{+(1)} \otimes_{A} m_{(0)} u_{+(2)} .
$$

The anti Yetter-Drinfel'd module $M$ is called stable (SaYD) if for all $m \in M$ one has

$$
m_{(0)} m_{(-1)}=m \text {. }
$$

Remark 2.8. Observe that it is not obvious that the expression on the right-hand side of (18) makes sense, but this follows from (1), (6), and (16).

\subsection{Cyclic (co)homology}

We recall that para-(co)cyclic $k$-modules generalise (co)cyclic $k$-modules by dropping the condition that the cyclic operator implements an action of $\mathbb{Z} /(n+1) \mathbb{Z}$ on the degree $n$ part. Thus a para-cyclic $k$-module is a simplicial $k$-module $\left(C_{\bullet}, d_{\bullet}, s_{\bullet}\right)$, and a para-cocyclic $k$-module is a cosimplicial $k$-module $\left(C^{\bullet}, \delta_{\bullet}, \sigma_{\bullet}\right)$ together with $k$-linear maps $t_{n}: C_{n} \rightarrow C_{n}$ and $\tau_{n}: C^{n} \rightarrow C^{n}$ satisfying, respectively

$$
\begin{aligned}
& d_{i} \circ t_{n}=\left\{\begin{array}{ll}
t_{n-1} \circ d_{i-1} & \text { if } 1 \leqslant i \leqslant n, \\
d_{n} & \text { if } i=0,
\end{array} \tau_{n} \circ \delta_{i}= \begin{cases}\delta_{i-1} \circ \tau_{n-1} & \text { if } 1 \leqslant i \leqslant n, \\
\delta_{n} & \text { if } i=0,\end{cases} \right. \\
& s_{i} \circ t_{n}=\left\{\begin{array}{ll}
t_{n+1} \circ s_{i-1} & \text { if } 1 \leqslant i \leqslant n, \\
t_{n+1}^{2} \circ s_{n} & \text { if } i=0,
\end{array} \tau_{n} \circ \sigma_{i}= \begin{cases}\sigma_{i-1} \circ \tau_{n+1} & \text { if } 1 \leqslant i \leqslant n, \\
\sigma_{n} \circ \tau_{n+1}^{2} & \text { if } i=0 .\end{cases} \right.
\end{aligned}
$$

It follows from these relations that $t_{n}^{n+1}$, respectively $\tau_{n}^{n+1}$, commutes with all the (co)faces and (co)degeneracies. Hence any para-(co)cyclic $k$-module defines a (co)cyclic one formed by the cokernels of $\operatorname{id}_{C_{n}}-t_{n}^{n+1}$, respectively the kernels of $\operatorname{id}_{C^{n}}-\tau_{n}^{n+1}$. 
The cyclic (co)homology of a para-(co)cyclic $k$-module is defined as the cyclic (co)homology of this associated (co)cyclic $k$-module.

Just like (co)cyclic $k$-modules, para-(co)cyclic ones can be viewed more conceptually as functors $\Lambda^{\text {op }} \rightarrow k$-Mod, respectively $\Lambda \rightarrow k$-Mod, where $\Lambda$ is the appropriate covering of Connes' cyclic category $\Lambda_{1}$. Hence, as Connes' category, $\Lambda$ has objects $\{[n]\}_{n \in \mathbb{N}}$, and the set of morphisms has generators obeying the same relations except for $\tau_{n}^{n+1}=\mathrm{id}_{[n]}$. The localisation of this category at the set of all $\tau_{n}$ has been studied already by Fergin and Tsygan in $[\mathbf{F T s}]$ where it is denoted by $\Lambda_{\infty}$. A general framework for such extensions of the simplicial category is provided by the concept of crossed simplicial groups due to Fiedorowicz and Loday [FiL]. However, we stress that in the present article $\tau_{n}$ is not assumed to be an isomorphism, so $\Lambda$ does not exactly arise from a crossed simplicial group but rather from a crossed simplicial semigroup. We will call $\Lambda$ the para-cyclic category.

\section{Hopf-cyclic cohomology with coefficients}

\subsection{Para-cocyclic structures on corings}

Following [Cr, BŞ2], in this section we first define an auxiliary para-cocyclic $k$-module that is relatively easy to construct. For this, $U$ just needs to be a left bialgebroid and $M$ needs to be a left $U$-comodule. Define then

$$
B^{\bullet}(U, M):=U^{\otimes_{A} \bullet+1} \otimes_{A^{e}} M,
$$

where $U$ is considered with the usual $(A, A)$-bimodule structure given by $\triangleright, \triangleleft$. So $B \bullet(U, M)$ is $\triangleright_{\triangleleft} U_{\triangleleft} \otimes_{A} \bullet+1 \otimes_{k} M$ modulo the span of elements

$$
\left\{u^{0} \otimes_{A} \cdots \otimes_{A} u^{n} \otimes_{A^{\mathrm{e}}} a m b-b \triangleright u^{0} \otimes_{A} \cdots \otimes_{A} u^{n} \triangleleft a \otimes_{A^{\mathrm{e}}} m \mid a, b \in A\right\} .
$$

Now define the following operators, where we abbreviate $w:=u^{0} \otimes_{A} \cdots \otimes_{A} u^{n}$ :

$$
\begin{aligned}
& \delta_{i}^{\prime}\left(w \otimes_{A^{\mathrm{e}}} m\right)= \begin{cases}u^{0} \otimes_{A} \cdots \otimes_{A} \Delta\left(u^{i}\right) \otimes_{A} \cdots \otimes_{A} u^{n} \otimes_{A^{\mathrm{e}}} m & \text { if } 0 \leqslant i \leqslant n, \\
u_{(2)}^{0} \otimes_{A} u^{1} \otimes_{A} \cdots \otimes_{A} m_{(-1)} u_{(1)}^{0} \otimes_{A^{\mathrm{e}}} m_{(0)} & \text { if } i=n+1,\end{cases} \\
& \sigma_{i}^{\prime}\left(w \otimes_{A^{\mathrm{e}}} m\right)=u^{0} \otimes_{A} \cdots \otimes_{A} t\left(\epsilon\left(u^{i+1}\right)\right) u^{i} \otimes_{A} \cdots \otimes_{A} u^{n} \otimes_{A^{\mathrm{e}}} m \quad 0 \leqslant i \leqslant n-1, \\
& \tau_{n}^{\prime}\left(w \otimes_{A^{\mathrm{e}}} m\right)=u^{1} \otimes_{A} \cdots \otimes_{A} u^{n} \otimes_{A} m_{(-1)} u^{0} \otimes_{A^{\mathrm{e}}} m_{(0)},
\end{aligned}
$$

which are shown to be well-defined using the Takeuchi condition for $\Delta_{M}$. The following is checked in a straightforward manner:

Lemma 3.1. The operators $\left(\delta_{\bullet}^{\prime}, \sigma_{\bullet}^{\prime}, \tau_{\bullet}^{\prime}\right)$ turn $B \bullet(U, M)$ into a para-cocyclic $k$-module.

3.2. The quotient $B^{\bullet}(U, M) \rightarrow C \cdot(U, M)$

The para-cocyclic $k$-module that defines Hopf-cyclic cohomology is the quotient

$$
U^{\otimes_{A} \bullet+1} \otimes_{U^{\text {op }}} M
$$

of $B \bullet(U, M)=U^{\otimes_{A} \bullet+1} \otimes_{A^{\mathrm{e}}} M$ defined above. This quotient makes sense whenever $M$ also carries a right $U$-module structure that induces the same $A^{\mathrm{e}}$-module structure as the left $U$-coaction; see (17). In the next section we will discuss that the para-cocyclic structure of $B^{\bullet}(U, M)$ descends to this quotient. However, for the applications in 
noncommutative geometry, one rewrites the resulting para-cocyclic $k$-module so that the object (but not the cocyclic operator) takes an easier form, and in the present section we construct the involved isomorphism.

Recall (e.g., from $[\mathbf{K o K r}$, Lem. 3]) that if $U$ is a left Hopf algebroid, then the tensor product $N \otimes_{A} M$ of $M \in U^{\text {op }}$-Mod, $N \in U$-Mod (considered with the $(A, A)$ bimodule structures (12) and (13)) carries a right $U$-module structure with action

$$
\left(n \otimes_{A} m\right) u:=u_{-} n \otimes_{A} m u_{+},
$$

and hence using (12) and (13) becomes an $(A, A)$-bimodule by

$$
a \bullet\left(n \otimes_{A} m\right) \triangleleft b:=(n \otimes m) t(a) s(b)=s(a) n \otimes_{A} m s(b)=a \triangleright n \otimes_{A} m \triangleleft b,
$$

where in the second equation (11) was used.

Now observe that on a right $U$-module of this form, the coinvariant functor

$$
-\otimes_{U} A: U^{\mathrm{op}}-\text { Mod } \rightarrow k \text {-Mod }
$$

takes a particularly simple form:

Lemma 3.2. If $U$ is a left Hopf algebroid, then for all $M \in U^{\mathrm{op}}$-Mod, $N \in U$-Mod there is a natural isomorphism $\left(N \otimes_{A} M\right) \otimes_{U} A \simeq N \otimes_{U^{\text {op }}} M$.

Proof. First write $A \otimes_{U^{\text {op }}}\left(N \otimes_{A} M\right)$ rather than $\left(N \otimes_{A} M\right) \otimes_{U} A$, and then apply the natural $k$-module isomorphism

$$
P \otimes_{U^{\text {op }}}\left(N \otimes_{A} M\right) \simeq\left(P \otimes_{A} N\right) \otimes_{U^{\text {op }}} M
$$

from [KoKr, Lem. 3] with $P=A$.

Note that $\left[\mathbf{K o K r}\right.$, Lem. 3] applied with $P=A, M=A^{\text {op }}$ yields the coinvariants in the form used in $[\mathbf{K o P}]$ where they were considered as a functor $U$-Mod $\rightarrow k$-Mod.

Applying Lemma 3.2 with $N=U^{\otimes_{A} \bullet+1}$ will lead to the simpler form of the paracocyclic $k$-module we are going to consider. To get there, we first remark:

Lemma 3.3. Let $M \in U^{\mathrm{op}}$-Mod and $N, P \in U$-Mod. Then one has

$$
\left(\text { un } \otimes_{A} p\right) \otimes_{U^{\mathrm{op}}} m=\left(n \otimes_{A} u_{-} p\right) \otimes_{U^{\mathrm{op}}} m u_{+}
$$

for all $m \in M, n \in N$, and $p \in P$.

Proof. One has

$$
\begin{aligned}
(\text { un } & \left.\otimes_{A} p\right) \otimes_{U^{\mathrm{op}}} m & & \\
& =\left(u_{+(1)} n \otimes_{A} u_{+(2)} u_{-} p\right) \otimes_{U^{\mathrm{op}}} m & & \text { by }(4), \\
& =u_{+}\left(n \otimes_{A} u_{-} p\right) \otimes_{U^{\mathrm{op}}} m & & \text { by the monoidal structure in } U \text {-Mod, } \\
& =\left(n \otimes_{A} u_{-} p\right) \otimes_{U^{\mathrm{op}}} m u_{+} . & &
\end{aligned}
$$

The well-definedness of the first operation follows from (11) using (12) and (13).

Using this we now obtain: 
Proposition 3.4. For $M \in U^{\mathrm{op}}-\mathrm{Mod}$ and $N \in U$-Mod, there is a canonical isomorphism of $k$-modules

$$
\phi:\left(U \otimes_{A} N\right) \otimes_{U^{\mathrm{op}}} M \stackrel{\simeq}{\longrightarrow} N \otimes_{A} M,
$$

given by

$$
\left(u \otimes_{A} n\right) \otimes_{U^{\mathrm{op}}} m \mapsto u_{-} n \otimes_{A} m u_{+} .
$$

Proof. The map $n \otimes_{A} m \mapsto\left(1 \otimes_{A} n\right) \otimes_{U^{\text {op }}} m$ is obviously a right inverse to (22), and by the preceding lemma it is also a left inverse.

In particular, this yields an isomorphism

$$
\phi: U^{\otimes_{A} \bullet+1} \otimes_{U^{\text {op }}} M \rightarrow U^{\otimes_{A}} \bullet \otimes_{A} M=: C \cdot(U, M),
$$

and the latter will be the ultimate object of study.

\subsection{Cyclic cohomology with coefficients for left Hopf algebroids}

Now we ask whether the para-cocyclic structure of $B \bullet(U, M)$ descends to $C \cdot(U, M)$. This is answered by a left Hopf algebroid left comodule and right module version of [BŞ2, Prop. 2.19], which generalises Proposition 5.2.1 in [Ko]:

Proposition 3.5. If $M$ is an anti Yetter-Drinfel'd module as in Definition 2.7, then the operators $\left(\delta_{\bullet}^{\prime}, \sigma_{\bullet}^{\prime}, \tau_{\bullet}^{\prime}\right)$ on $B^{\bullet}(U, M)$ from (20) descend to well-defined operators on $U^{\otimes_{A} \bullet+1} \otimes_{U^{\text {op }}} M$.

Proof. One needs to prove that the operators $\left(\delta_{\bullet}^{\prime}, \sigma_{\bullet}^{\prime}, \tau_{\bullet}^{\prime}\right)$ are $U^{\text {op }}$-balanced, i.e., that one has, for example,

$$
\tau_{n}^{\prime}\left(u^{0} \otimes_{A} \cdots \otimes_{A} u^{n} \otimes_{U^{\mathrm{op}}} m v\right)=\tau_{n}^{\prime}\left(v_{(1)} u^{0} \otimes_{A} \cdots \otimes_{A} v_{(n+1)} u^{n} \otimes_{U^{\mathrm{op}}} m\right)
$$

for any $v \in U$. This is shown by expressing the right-hand side as

$$
\begin{aligned}
v_{(2)} & u^{1} \otimes_{A} \cdots \otimes_{A} v_{(n+1)} u^{n} \otimes_{A} m_{(-1)} v_{(1)} u^{0} \otimes_{U^{\mathrm{op}}} m_{(0)} \\
& =v_{(2)} u^{1} \otimes_{A} \cdots \otimes_{A} v_{(n+1)} u^{n} \otimes_{A} \operatorname{se}\left(v_{(n+2)}\right) m_{(-1)} v_{(1)} u^{0} \otimes_{U^{\mathrm{op}}} m_{(0)} \\
& =v_{(2)} u^{1} \otimes_{A} \cdots \otimes_{A} v_{(n+1)} u^{n} \otimes_{A} v_{(n+2)+} v_{(n+2)-} m_{(-1)} v_{(1)} u^{0} \otimes_{U^{\mathrm{op}}} m_{(0)} \\
& =v_{+(2)} u^{1} \otimes_{A} \cdots \otimes_{A} v_{+(n+1)} u^{n} \otimes_{A} v_{+(n+2)} v_{-} m_{(-1)} v_{+(1)} u^{0} \otimes_{U^{\mathrm{op}}} m_{(0)} \\
& =u^{1} \otimes_{A} \cdots \otimes_{A} u^{n} \otimes_{A} v_{-} m_{(-1)} v_{+(1)} u^{0} \otimes_{U^{\mathrm{op}}} m_{(0)} v_{+(2)} \\
& =u^{1} \otimes_{A} \cdots \otimes_{A} u^{n} \otimes_{A}(m v)_{(-1)} u^{0} \otimes_{U^{\mathrm{op}}}(m v)_{(0)},
\end{aligned}
$$

which is the left-hand side. Here we used the counital identities of the left coproduct in the second line, (10) in the third line, (7) combined with (higher) coassociativity in the fourth line, and finally the anti Yetter-Drinfel'd condition (18). Similar calculations can be made for the cofaces and codegeneracies.

We denote the resulting para-cocyclic structure on $C \cdot(U, M)$ by

$$
\begin{aligned}
\delta_{i} & :=\phi \circ \bar{\delta}_{i}^{\prime} \circ \phi^{-1}, \\
\sigma_{i} & :=\phi \circ \bar{\sigma}_{i}^{\prime} \circ \phi^{-1}, \\
\tau_{i} & :=\phi \circ \bar{\tau}_{i}^{\prime} \circ \phi^{-1},
\end{aligned}
$$

where $\phi$ is as in (23), and $\bar{\delta}_{i}^{\prime}, \bar{\sigma}_{j}^{\prime}, \bar{\tau}_{n}^{\prime}$ are the para-cocyclic operators on $U^{\otimes_{A} \bullet+1} \otimes_{U^{\text {op }}} M$ that descend from $B \bullet(U, M)$. 
A short computation yields the explicit expressions given in Theorem 1.1:

$$
\begin{gathered}
\delta_{i}\left(z \otimes_{A} m\right)= \begin{cases}1 \otimes_{A} u^{1} \otimes_{A} \cdots \otimes_{A} u^{n} \otimes_{A} m & \text { if } i=0, \\
u^{1} \otimes_{A} \cdots \otimes_{A} \Delta\left(u^{i}\right) \otimes_{A} \cdots \otimes_{A} u^{n} \otimes_{A} m & \text { if } 1 \leqslant i \leqslant n, \\
u^{1} \otimes_{A} \cdots \otimes_{A} u^{n} \otimes_{A} m_{(-1)} \otimes_{A} m_{(0)} & \text { if } i=n+1,\end{cases} \\
\delta_{j}(m)= \begin{cases}1 \otimes_{A} m & \text { if } j=0, \\
m_{(-1)} \otimes_{A} m_{(0)} & \text { if } j=1,\end{cases} \\
\sigma_{i}\left(z \otimes_{A} m\right)=u^{1} \otimes_{A} \cdots \otimes_{A} \epsilon\left(u^{i+1}\right) \otimes_{A} \cdots \otimes_{A} u^{n} \otimes_{A} m \\
\tau_{n}\left(z \otimes_{A} m\right)=u_{-(1)}^{1} u^{2} \otimes_{A} \cdots \otimes_{A} u_{-(n-1)}^{1} u^{n} \otimes_{A} u_{-(n)}^{1} m_{(-1)} \otimes_{A} m_{(0)} u_{+}^{1},
\end{gathered}
$$

where we abbreviate $z:=u^{1} \otimes_{A} \cdots \otimes_{A} u^{n}$.

In this form, the well-definedness and the well-definedness over the Sweedler presentations of these operators can be seen directly (using (11) as well as the Takeuchi properties of $\Delta$ and $\Delta_{M}$ ). Observe, however, that the condition $m a=m s(a)$ from (17) is not needed to make the operators (24) well-defined and well-defined over the Sweedler presentation, but only to give a sense to the above quotienting process.

It is less obvious that the stability condition on $M$ implies cyclicity. This is, however, immediate from the presentation of $C \bullet(U, M)$ as a quotient of $B \bullet(U, M)$ :

Theorem 3.6. If $U$ is a left Hopf algebroid and $M$ is a stable anti Yetter-Drinfel'd module, then $\left(C^{\bullet}(U, M), \delta_{\bullet}, \sigma_{\bullet}, \tau_{\bullet}\right)$ is a cocyclic $k$-module.

Proof. By its construction, $\left(C_{\bullet}(U, M), \delta_{\bullet}, \sigma_{\bullet}, \tau_{\bullet}\right)$ is a para-cocyclic object and as such is isomorphic to $\left(U^{\otimes_{A} \bullet+1} \otimes_{U^{\text {op }}} M, \bar{\delta}_{\bullet}^{\prime}, \bar{\sigma}_{\bullet}^{\prime}, \bar{\tau}_{\bullet}^{\prime}\right)$ obtained in Proposition 3.5. It remains to show that this quotient of $B^{\bullet}(U, M)$ is cocyclic if $M$ is stable:

$$
\begin{aligned}
\left(\bar{\tau}_{n}^{\prime}\right)^{n+1}\left(u^{0} \otimes_{A} \cdots \otimes_{A} u^{n} \otimes_{U^{\mathrm{op}}} m\right) & =m_{(-n-1)} u^{0} \otimes_{A} \cdots \otimes_{A} m_{(-1)} u^{n} \otimes_{U^{\mathrm{op}}} m_{(0)} \\
& =m_{(-1)} \cdot\left(u^{0} \otimes_{A} \cdots \otimes_{A} u^{n}\right) \otimes_{U^{\mathrm{op}}} m_{(0)} \\
& =u^{0} \otimes_{A} \cdots \otimes_{A} u^{n} \otimes_{U^{\mathrm{op}}} m_{(0)} m_{(-1)},
\end{aligned}
$$

where $\cdot$ denotes the diagonal left $U$-action via the left coproduct.

By the last line in the proof of the preceding theorem, one may be tempted to think that an aYD module defines a para-cocyclic module which is cocyclic if $M$ is stable. The observation we add here is that for defining a para-cocyclic module the aYD property (18), i.e., compatibility between $U$-action and $U$-coaction, is not required:

Theorem 3.7. Let $U$ be a left Hopf algebroid and $M$ a right $U$-module and left $U$-comodule, and let the respective left A-actions be compatible in the following sense:

$$
a m=a \bullet m, \quad m \in M, a \in A .
$$

Then $\left(C \bullet(U, M), \delta_{\bullet}, \sigma_{\bullet}, \tau_{\bullet}\right)$ is a para-cocyclic $k$-module.

Proof. We need to check the relations in the right column in (19). Since we do not assume that $M$ is aYD here, i.e., compatibility between action and coaction, the only relations that need to be checked are those that have the $U$-action on $M$ followed by 
an operation involving the $U$-coaction on $M$. Here, this is only $\tau_{n} \circ \sigma_{0}=\sigma_{n} \circ \tau_{n+1}^{2}$, which is proven as follows: first compute

$$
\begin{aligned}
\sigma_{n} & \left(\tau_{n+1}\left(u^{1} \otimes_{A} \cdots \otimes_{A} u^{n+1} \otimes_{A} m\right)\right) \\
& =\sigma_{n}\left(u_{-(1)}^{1} u^{2} \otimes_{A} \cdots \otimes_{A} u_{-(n)}^{1} u^{n+1} \otimes_{A} u_{-(n+1)}^{1} m_{(-1)} \otimes_{A} m_{(0)} u_{+}^{1}\right) \\
& =u_{-(1)}^{1} u^{2} \otimes_{A} \cdots \otimes_{A} t\left(\epsilon\left(u_{-(n+1)}^{1} m_{(-1)}\right)\right) u_{-(n)}^{1} u^{n+1} \otimes_{A} m_{(0)} u_{+}^{1} \\
& =u_{-(1)}^{1} u^{2} \otimes_{A} \cdots \otimes_{A} u_{-(n)}^{1} u^{n+1} \otimes_{A} m_{(0)} t\left(\epsilon\left(m_{(-1)}\right)\right) u_{+}^{1} \\
& =u_{-(1)}^{1} u^{2} \otimes_{A} \cdots \otimes_{A} u_{-(n)}^{1} u^{n+1} \otimes_{A} m u_{+}^{1},
\end{aligned}
$$

where we used the Takeuchi property (6) in the fourth line and (25) together with the comodule properties in the fifth, so that terms involving the coaction disappear. Hence

$$
\begin{aligned}
& \sigma_{n} \tau_{n+1}^{2}\left(u^{1} \otimes_{A} \cdots \otimes_{A} u^{n+1} \otimes_{A} m\right) \\
& =\sigma_{n} \tau_{n+1}\left(u_{-(1)}^{1} u^{2} \otimes_{A} \cdots \otimes_{A} u_{-(n)}^{1} u^{n+1} \otimes_{A} u_{-(n+1)}^{1} m_{(-1)} \otimes_{A} m_{(0)} u_{+}^{1}\right) \\
& =\left(u_{-(1)}^{1} u^{2}\right)_{-(1)} u_{-(2)}^{1} u^{3} \otimes_{A} \cdots \otimes_{A}\left(u_{-(1)}^{1} u^{2}\right)_{-(n)} u_{-(n+1)}^{1} m_{(-1)} \otimes_{A} m_{(0)} u_{+}^{1}\left(u_{-(1)}^{1} u^{2}\right)_{+} \\
& =u_{-(1)}^{2}\left(\left(u_{-}^{1}\right)_{(1)-}\left(u_{-}^{1}\right)_{(2)}\right)_{(1)} u^{3} \otimes_{A} \cdots \\
& \quad \otimes_{A} u_{-(n)}^{2}\left(\left(u_{-}^{1}\right)_{(1)-}\left(u_{-}^{1}\right)_{(2)}\right)_{(n)} m_{(-1)} \otimes_{A} m_{(0)} u_{+}^{1}\left(u_{-}^{1}\right)_{(1)+} u_{+}^{2} \\
& =u_{-(1)}^{2} u^{3} \otimes_{A} \cdots \otimes_{A} u_{(n-1)}^{2} u^{n+1} \otimes_{A} u_{-(n)}^{2} m_{(-1)} \otimes_{A} m_{(0)} s\left(\epsilon\left(u^{1}\right)\right) u_{+}^{2},
\end{aligned}
$$

where in the fifth line (5) was used and (10) in the sixth. By (11) this is now easily seen to be equal to $\tau_{n} \sigma_{0}\left(u^{1} \otimes_{A} \cdots \otimes_{A} u^{n+1} \otimes_{A} m\right)$.

Definition 3.8. For a right $U$-module left $U$-comodule $M$ with compatible induced left $A$-actions over a left Hopf algebroid $U$, we denote by $H^{\bullet}(U, M)$ and $H C \bullet(U, M)$ the simplicial and cyclic cohomology groups of $C \cdot(U, M)$. We refer to $H C \bullet(U, M)$ as to the Hopf-cyclic cohomology of $U$ with coefficients in $M$.

Note that the simplicial cohomology is the ordinary Cotor over $U$ :

Proposition 3.9 ([Ko, $\mathbf{K o P}])$. If $U_{\triangleleft}$ is flat as a right $A$-module, then one has

$$
H^{\bullet}(U, M) \simeq \operatorname{Cotor}_{U}^{\bullet}(A, M) .
$$

Remark 3.10. If $U$ is a (full) Hopf algebroid over base algebras $A$ and $B \simeq A^{\text {op }}$, it is easy to check that $B$ fulfills the properties of an anti Yetter-Drinfel'd module with respect to the right $U$-action given by the right counit of the underlying right bialgebroid. This module is stable if the antipode of the Hopf algebroid is an involution. The operators (24) reduce here to the well-known Hopf-cyclic operators for Hopf algebroids, cf. [CM1, KhR, Ko, KoP]. For example, the cyclic operator reduces in such a case to

$$
\tau_{n}\left(h^{1} \otimes_{A} \cdots \otimes_{A} h^{n}\right)=\left(S\left(h^{1}\right)\right)_{(1)} h^{2} \otimes_{A} \cdots \otimes_{A}\left(S\left(h^{1}\right)\right)_{(n-1)} h^{n} \otimes_{A}\left(S\left(h^{1}\right)\right)_{(n)} .
$$

\section{Hopf-cyclic homology with coefficients}

\subsection{Cyclic homology with coefficients for left Hopf algebroids}

Let $U$ be a left Hopf algebroid over $A$ with structure maps as before, and let $M$ be a left $U$-comodule with left coaction denoted $\Delta_{M}: m \mapsto m_{(-1)} \otimes_{A} m_{(0)}$ with underlying 
left $A$-action $(a, m) \mapsto a m$, and simultaneously a right $U$-module with right action denoted $(m, u) \mapsto m u$, subject to the compatibility condition (17) with respect the two induced $A^{\mathrm{e}}$-module structures.

Now define

$$
C .(U, M):=M \otimes_{A^{\mathrm{op}}}\left(\triangleleft U_{\triangleleft}\right)^{\otimes_{A^{\mathrm{op}}}},
$$

where the tensor product is formed as in (3). On $C_{\bullet}(U, M)$, define the following operators, abbreviating $x:=u^{1} \otimes_{A^{\text {op }}} \cdots \otimes_{A^{\text {op }}} u^{n}$ :

$$
\begin{aligned}
& d_{i}\left(m \otimes_{A^{\mathrm{op}}} x\right)= \begin{cases}m \otimes_{A^{\mathrm{op}}} u^{1} \otimes_{A^{\mathrm{op}}} \cdots \otimes_{A^{\mathrm{op}}} \epsilon\left(u^{n}\right) u^{n-1} & \text { if } i=0, \\
m \otimes_{A^{\mathrm{op}}} \cdots \otimes_{A^{\mathrm{op}}} u^{n-i} u^{n-i+1} \otimes_{A^{\mathrm{op}}} \cdots & \text { if } 1 \leqslant i \leqslant n-1, \\
m u^{1} \otimes_{A^{\mathrm{op}}} u^{2} \otimes_{A^{\mathrm{op}}} \cdots \otimes_{A^{\mathrm{op}}} u^{n} & \text { if } i=n,\end{cases} \\
& s_{i}\left(m \otimes_{A^{\mathrm{op}}} x\right)= \begin{cases}m \otimes_{A^{\mathrm{op}}} u^{1} \otimes_{A^{\mathrm{op}}} \cdots \otimes_{A^{\mathrm{op}}} u^{n} \otimes_{A^{\mathrm{op}}} 1 & \text { if } i=0, \\
m \otimes_{A^{\mathrm{op}}} \cdots \otimes_{A^{\mathrm{op}}} u^{n-i} \otimes_{A^{\mathrm{op}}} 1 \otimes_{A^{\mathrm{op}}} u^{n-i+1} \otimes_{A^{\mathrm{op}}} \cdots & \text { if } 1 \leqslant i \leqslant n-1, \\
m \otimes_{A^{\mathrm{op}}} 1 \otimes_{A^{\mathrm{op}}} u^{1} \otimes_{A^{\mathrm{op}}} \cdots \otimes_{A^{\mathrm{op}}} u^{n} & \text { if } i=n,\end{cases} \\
& t_{n}\left(m \otimes_{A^{\mathrm{op}}} x\right)=m_{(0)} u_{+}^{1} \otimes_{A^{\mathrm{op}}} u_{+}^{2} \otimes_{A^{\mathrm{op}}} \cdots \otimes_{A^{\mathrm{op}}} u_{+}^{n} \otimes_{A^{\mathrm{op}}} u_{-}^{n} \cdots u_{-}^{1} m_{(-1)} .
\end{aligned}
$$

Elements of degree zero (i.e., of $M)$ are mapped to zero by the face maps, $d_{0}(m)$ $=0$ for all $m \in M$. Well-definedness and well-definedness over the various Sweedler presentations follow from (6), (11), (15) and (16). Similarly, as in the cohomology case, these operators still make sense if one drops the condition $m a=m s(a)$ from the axiom (17) as well as the aYD condition (18).

As one might expect, we will obtain dually to Theorems 3.6 and 3.7:

Theorem 4.1. Let $U$ be a left Hopf algebroid.

1. If $M$ is a right $U$-module and left $U$-comodule with respective left A-actions compatible as in (25), then $\left(C_{\bullet}(U, M), d_{\bullet}, s_{\bullet}, t_{\bullet}\right)$ is a para-cyclic $k$-module.

2. If $M$ is even a stable anti Yetter-Drinfel'd module, then $\left(C \cdot(U, M), d_{\bullet}, s_{\bullet}, t_{\bullet}\right)$ is a cyclic $k$-module.

We will prove this below by presenting $C \cdot(U, M)$ as a cyclic dual of $C \bullet(U, M)$.

Definition 4.2. For a right $U$-module left $U$-comodule $M$ with compatible induced left $A$-actions over a left Hopf algebroid $U$, we denote by $H_{\bullet}(U, M)$ and $H C$. $(U, M)$ the simplicial and cyclic homology groups of $C \cdot(U, M)$. We refer to $H C \cdot(U, M)$ as to the Hopf-cyclic homology of $U$ with coefficients in $M$.

Dually to Proposition 3.9, one has:

Proposition $4.3([\mathbf{K o}, \mathbf{K o P}])$. If $\checkmark U$ is projective as a left $A$-module, then one has

$$
H_{\bullet}(U, M) \simeq \operatorname{Tor}_{\bullet}^{U}(M, A) .
$$

Remark 4.4. As in Remark 3.10, in a full Hopf algebroid $H$ the base algebra $B$ of the underlying right bialgebroid is an anti Yetter-Drinfel'd module which is stable if the antipode is an involution. The cyclic operator assumes the form

$$
t_{n}\left(u_{1} \otimes_{A^{\mathrm{op}}} \cdots \otimes_{A^{\mathrm{op}}} u_{n}\right)=u_{2}^{(1)} \otimes_{A^{\mathrm{op}}} \cdots \otimes_{A^{\mathrm{op}}} u_{n}^{(1)} \otimes_{A^{\mathrm{op}}} S\left(u_{1} u_{2}^{(2)} \cdots u_{n}^{(2)}\right),
$$

where the Sweedler superscripts refer to the right coproduct. This is the same expression as the inverse of the cyclic operator from $[\mathbf{K o}, \mathbf{K o P}]$; see our explanations below. 


\subsection{Cyclic duality [C, E, FTs, L]}

Recall that the cyclic category is self-dual, that is, we have $\Lambda_{1} \simeq \Lambda_{1}^{\mathrm{op}}$, and therefore cocyclic $k$-modules and cyclic $k$-modules can be canonically identified. However, there are even infinitely many such canonical identifications since the cyclic category has many autoequivalences (see e.g., [L, 6.1.14 and E.6.1.5], but note that the very last line of $[\mathbf{L}, 6.1 .14]$ should read $\left.\tau_{n} \mapsto \tau_{n}^{-1}\right)$.

Fergin and Tsygan have generalised the duality to their category $\Lambda_{\infty}$, that is, to para-(co)cyclic $k$-modules whose cyclic operators are isomorphisms (see [FTs, $§ \mathrm{~A} 7]$ ). Unfortunately, they use the most common choice of equivalence $\Lambda_{\infty} \simeq \Lambda_{\infty}^{\mathrm{op}}$ which does not extend to general para-(co)cyclic objects.

However, a different equivalence $\Lambda_{\infty} \simeq \Lambda_{\infty}^{\text {op }}$ does lift to a functor $\Lambda^{\text {op }} \rightarrow \Lambda$, so that one can assign a para-cyclic module to any para-cocyclic module even with not necessarily invertible $\tau_{n}$; one only has to bear in mind that this process is in general not invertible. Still, it can be applied in full generality to the para-cocyclic object $C \cdot(U, M)$, even when $M$ is not SaYD, and hence Theorem 4.1 follows from the results of the previous section.

Explicitly, we use the following convention for this functor. We decided to stick to the term "cyclic dual" although it is no longer a true duality in general:

Definition 4.5. The cyclic dual of a para-cocyclic $k$-module $C^{\bullet}=\left(C^{\bullet}, \delta_{\bullet}, \sigma_{\bullet}, \tau_{\bullet}\right)$ is the cyclic $k$-module $C_{\bullet}:=\left(C_{\bullet}, d_{\bullet}, s_{\bullet}, t_{\bullet}\right)$, where $C_{n}:=C^{n}$, and

$$
\begin{array}{lll}
d_{i}:=\sigma_{n-(i+1)}: C_{n} \rightarrow C_{n-1}, & 0 \leqslant i<n, \\
d_{n}:=\sigma_{n-1} \circ \tau_{n}: C_{n} \rightarrow C_{n-1}, & \\
s_{i}:=\delta_{n-(i+1)}: C_{n-1} \rightarrow C_{n}, & 0 \leqslant i<n, \\
t_{n}:=\tau_{n} \quad: C_{n} \rightarrow C_{n} . &
\end{array}
$$

For the convenience of the reader we verify at least some of the relations:

Lemma 4.6. The cyclic dual of any para-cocyclic $k$-module is a para-cyclic $k$-module.

Proof. We need to check the para-cyclic relations by using the para-cocyclic ones, which is straightforward. For example, let $i<j$ and $j<n$; then $n-(i+2) \geqslant n-$ $(j+1)$, and

$$
d_{i} \circ d_{j}=\sigma_{(n-1)-(i+1)} \circ \sigma_{n-(j+1)}=\sigma_{(n-1)-j} \circ \sigma_{n-(i+1)}=d_{j-1} \circ d_{i} .
$$

For $j=n$ (in which case $i \leqslant n-2$ ),

$$
\begin{aligned}
d_{i} \circ d_{n} & =\sigma_{(n-1)-(i+1)} \circ \sigma_{n-1} \circ \tau_{n} \\
& =\sigma_{n-2} \circ \sigma_{(n-1)-(i+1)} \circ \tau_{n} \\
& =\sigma_{n-2} \circ \tau_{n-1} \circ \sigma_{n-(i+1)}=d_{n-1} \circ d_{i} .
\end{aligned}
$$

Likewise,

$$
d_{i} \circ s_{i}=\sigma_{n-(i+1)} \circ \delta_{n-(i+1)}=\mathrm{id}=\sigma_{n-j-2} \circ \delta_{n-j-1}=d_{j+1} \circ s_{j} .
$$

Also,

$$
d_{i} \circ t_{n}=\sigma_{n-(i+1)} \circ \tau_{n}=\sigma_{n-i-1} \circ \tau_{n}=\tau_{n-1} \circ \sigma_{n-i}=t_{n-1} \circ d_{i-1}
$$

for $1 \leqslant i \leqslant n-1$, and for $i=n$ the identity $d_{0} \circ t_{n}=d_{n}$ is trivially fulfilled. Finally,

$$
s_{0} \circ t_{n}=\delta_{n-1} \circ \tau_{n}=\tau_{n+1} \circ \delta_{n}=\tau_{n+1} \circ \tau_{n+1} \delta_{0}=t_{n+1}^{2} \circ s_{n} .
$$


The rest of the simplicial and cyclic identities are left to the reader.

Remark 4.7. Note that the last coface map $\delta_{n}: C^{n-1} \rightarrow C^{n}$ is not used in the construction of the cyclic dual: there is one less degeneracy $s_{i}: C_{n-1} \rightarrow C_{n}$ than there are cofaces $\delta_{i}: C^{n-1} \rightarrow C^{n}$. Conversely, there are not enough codegeneracies to derive all the face maps: the last face map $d_{n}$ uses the extra codegeneracy $\sigma_{n-1} \circ \tau_{n}$ that arises from the (para-)cocyclic operator.

Remark 4.8. Observe that the cyclic homology of the cyclic dual of a given cocyclic $k$-module is independent of the choice of the self-duality of the cyclic category $\Lambda_{1}$. This follows from the description of cyclic homology as $\operatorname{Tor}_{\bullet}^{\Lambda_{1}^{\mathrm{op}}}(k, C)$ (cf. [L, Thm. 6.2.8]) in combination with the fact that all autoequivalences of $\Lambda_{1}$ leave the trivial cyclic $k$-module $k$ invariant.

Remark 4.9. Two relatively straightforward cases in which the cyclic operator is not invertible are that of a Hopf algebra $U$ (over $A=k$ ) whose antipode is not bijective, taking the coefficients to be $M=k$ with trivial action $1 \cdot u=\epsilon(u)$ and trivial coaction $\Delta_{M}(1)=1 \otimes 1$, or that of $U=A^{e}, M=A_{\sigma}$, discussed in Section 5.2 below, when $\sigma$ is not bijective.

However, it seems worthwhile to remark that $\tau$ is invertible if $U$ is a full Hopf algebroid with invertible antipode $S$ and $M$ has yet some additional structure: recall first [B1] that the two constituting bialgebroids (i.e., left and right) in a full Hopf algebroid have different underlying corings (over anti-isomorphic base algebras) that have a priori different categories of comodules. A Hopf algebroid (say, left) comodule is then, roughly speaking, both a left and a right bialgebroid (left) comodule, the two structures being compatible with each other. If $M$ is a left comodule over the full Hopf algebroid $U$ and aYD, in the sense of Definition 2.7 with respect to the underlying left bialgebroid, one verifies by a tedious but straightforward induction on $n$ that

$$
\begin{aligned}
& \tau_{n}^{-1}\left(w \otimes_{A} m\right) \\
& \quad:=\left(S^{-2}\left(u_{-}^{n}\right) m^{(-1)}{ }_{(1)}\right) \cdot\left(1 \otimes_{A} u^{1} \otimes_{A} \cdots \otimes_{A} u^{n-1}\right) \otimes_{A} m^{(0)} S^{-1}\left(m^{(-1)}{ }_{(2)}\right) S^{-2}\left(u_{+}^{n}\right)
\end{aligned}
$$

yields an inverse for the cocyclic operator $\tau_{n}$ from (24), where we abbreviated $w:=u^{1} \otimes_{A} \cdots \otimes_{A} u^{n}$. Here $\cdot$ denotes the diagonal action via the left coproduct and Sweedler superscripts the left coaction with respect to the underlying right bialgebroid in $U$. In case $M=B \simeq A^{\text {op }}$, this reduces to the well-known expression

$$
u^{1} \otimes_{A} \cdots \otimes_{A} u^{n} \mapsto\left(S^{-1}\left(u^{n}\right)\right) \cdot\left(1 \otimes_{A} u^{1} \otimes_{A} \cdots \otimes_{A} u^{n-1}\right)
$$

from $[\mathbf{K o}, \mathbf{K o P}]$. If $M$ is an SaYD so that $C_{\bullet}(U, M)$ is cocyclic, then the inverse of $\tau_{n}$ is simply given for any left Hopf algebroid $U$ by

$$
\tau_{n}^{-1}\left(u^{1} \otimes_{A} \cdots \otimes_{A} u^{n} \otimes_{A} m\right)=u_{-}^{n} m_{(-1)} \cdot\left(1 \otimes_{A} u^{1} \otimes_{A} \cdots \otimes_{A} u^{n-1}\right) \otimes_{A} m_{(0)} u_{+}^{n} .
$$

\subsection{The Hopf-Galois map and cyclic duality}

The explicit map implementing the isomorphism $C \cdot(U, M) \simeq C_{\bullet}(U, M)$ is given by generalising the Hopf-Galois map (2): 
Lemma 4.10. For each $n \geqslant 0$, the $k$-modules $C_{n}(U, M)$ and $C^{n}(U, M)$ are isomorphic by means of the Hopf-Galois map $\varphi_{n}: C_{n}(U, M) \rightarrow C^{n}(U, M)$ in degree $n$, defined by $\varphi_{0}:=\operatorname{id}_{M}, \varphi_{1}: m \otimes_{A^{\text {op }}} u \mapsto u \otimes_{A} m$, and for $n \geqslant 2$

$$
\begin{aligned}
\varphi_{n}\left(m \otimes_{A^{\mathrm{op}}} u^{1} \otimes_{A^{\mathrm{op}}} \cdots \otimes_{A^{\mathrm{op}}} u^{n}\right) \\
\quad:=u_{(1)}^{1} \otimes_{A} u_{(2)}^{1} u_{(1)}^{2} \otimes_{A} \cdots \otimes_{A} u_{(n)}^{1} u_{(n-1)}^{2} \cdots u_{(2)}^{n-1} u^{n} \otimes_{A} m,
\end{aligned}
$$

with inverse

$$
\begin{aligned}
& \psi_{n}\left(u^{1} \otimes_{A} \cdots \otimes_{A} u^{n} \otimes_{A} m\right) \\
& \quad:=m \otimes_{A^{\mathrm{op}}} u_{+}^{1} \otimes_{A^{\mathrm{op}}} u_{-}^{1} u_{+}^{2} \otimes_{A^{\mathrm{op}}} u_{-}^{2} u_{+}^{3} \otimes_{A^{\mathrm{op}}} \cdots \otimes_{A^{\mathrm{op}}} u_{-}^{n-1} u^{n} .
\end{aligned}
$$

Proof. Well-definedness and well-definedness over the respective Sweedler presentations follow from the Takeuchi conditions (1) and (6). The fact that $\varphi$ and $\psi$ are mutually inverse is verified by induction on $n$ using the properties (4) and (5).

Lemma 4.11. If $U$ is a left Hopf algebroid, then the Hopf-Galois map identifies $C_{\bullet}(U, M)$ with the cyclic dual of the cocyclic module $C^{\bullet}(U, M)$ of Theorem 3.6.

Proof. We need to show, e.g., for the cyclic operators (24) and (26)

$$
\tau_{n} \circ \varphi_{n}=\varphi_{n} \circ t_{n}
$$

with respect to the map (27). This is a straightforward verification: one has

$$
\begin{aligned}
& \tau_{n} \varphi_{n}\left(m \otimes_{A^{\mathrm{op}}} u^{1} \otimes_{A^{\mathrm{op}}} \cdots \otimes_{A^{\mathrm{op}}} u^{n}\right) \\
& =\tau_{n}\left(u_{(1)}^{1} \otimes_{A} u_{(2)}^{1} u_{(1)}^{2} \otimes_{A} \cdots \otimes_{A} u_{(n)}^{1} u_{(n-1)}^{2} \cdots u_{(2)}^{n-1} u^{n} \otimes_{A} m\right) \\
& =u_{(1)-(1)}^{1} u_{(2)}^{1} u_{(1)}^{2} \otimes_{A} \cdots \otimes_{A} u_{(1)-(n-1)}^{1} u_{(n)}^{1} u_{(n-1)}^{2} \cdots u_{(2)}^{n-1} u^{n} \\
& \otimes_{A} u_{(1)-(n)}^{1} m_{(-1)} \otimes_{A} m_{(0)} u_{(1)+}^{1} \\
& =u_{(1)-(1)}^{1} u_{(2)}^{1} u_{(1)}^{2} \otimes_{A} \cdots \otimes_{A} u_{(1)-(n-1)}^{1} u_{(n)}^{1} u_{(n-1)}^{2} \cdots u_{(2)}^{n-1} u^{n} \\
& \otimes_{A} u_{(1)+-}^{1} m_{(-1)} \otimes_{A} m_{(0)} u_{(1)++}^{1} \\
& =u_{(1)}^{2} \otimes_{A} u_{(2)}^{2} u_{(1)}^{3} \otimes_{A} \cdots \otimes_{A} u_{(n-1)}^{2} \cdots u_{(2)}^{n-1} u^{n} \otimes_{A} u_{-}^{1} m_{(-1)} \otimes_{A} m_{(0)} u_{+}^{1}
\end{aligned}
$$

using (5) and (8), whereas

$$
\begin{aligned}
& \varphi_{n} t_{n}\left(m \otimes_{A^{\mathrm{op}}} u^{1} \otimes_{A^{\mathrm{op}}} \cdots \otimes_{A^{\mathrm{op}}} u^{n}\right) \\
& =\varphi_{n}\left(m_{(0)} u_{+}^{1} \otimes_{A^{\mathrm{op}}} u_{+}^{2} \otimes_{A^{\mathrm{op}}} \cdots \otimes_{A^{\mathrm{op}}} u_{+}^{n} \otimes_{A^{\mathrm{op}}} u_{-}^{n} \cdots u_{-}^{1} m_{(-1)}\right) \\
& =u_{+(1)}^{2} \otimes_{A} u_{+(2)}^{2} u_{+(1)}^{3} \otimes_{A} \cdots \otimes_{A} u_{+(n)}^{2} u_{+(n-1)}^{3} \cdots u_{+(2)}^{n} u_{-}^{n} \cdots u_{-}^{1} m_{(-1)} \otimes_{A} m_{(0)} u_{+}^{1} \\
& =u_{(1)}^{2} \otimes_{A} u_{(2)}^{2} u_{(1)}^{3} \otimes_{A} \cdots \otimes_{A} u_{(n-1)}^{2} \cdots u_{(2)}^{n-1} u^{n} \otimes_{A} u_{-}^{1} m_{(-1)} \otimes_{A} m_{(0)} u_{+}^{1}
\end{aligned}
$$

by (4) and (7), and the claim follows. The corresponding identities relating (co)faces to (co)degeneracies are left to the reader.

Proof of Theorem 4.1. This now follows from Theorem 3.6 and Theorem 3.7.

\section{Examples}

\subsection{Lie-Rinehart homology with coefficients}

Let $(A, L)$ be a Lie-Rinehart algebra over a commutative $k$-algebra $A$ and $V L$ be its universal enveloping algebra (see $[\mathbf{R i}]$ ). 
The left Hopf algebroid structure of $V L$ has been described in [KoKr $]$; as therein, we denote by the same symbols elements $a \in A$ and $X \in L$ and the corresponding generators in $V L$. The maps $s=t$ are equal to the canonical injection $A \rightarrow V L$. The coproduct and the counit are given by

$$
\begin{array}{rlrl}
\Delta(X) & :=X \otimes_{A} 1+1 \otimes_{A} X, & & \epsilon(X):=0, \\
\Delta(a) & :=a \otimes_{A} 1, & \epsilon(a):=a,
\end{array}
$$

whereas the inverse of the Hopf-Galois map is

$$
X_{+} \otimes_{A^{\mathrm{op}}} X_{-}:=X \otimes_{A^{\mathrm{op}}} 1-1 \otimes_{A^{\mathrm{op}}} X, \quad a_{+} \otimes_{A^{\mathrm{op}}} a_{-}:=a \otimes_{A} 1 .
$$

By universality, these maps can be extended to $V L$.

Recall from [Hue] that a right $(A, L)$-module $M$ is simultaneously a left $A$-module with action $(a, m) \mapsto a m$ and a right $L$-module with action $(m, X) \mapsto m X$, subject to the compatibility conditions

$$
\begin{aligned}
& (a m) X=a(m X)-X(a) m, \quad m \in M, a \in A, X \in L . \\
& m(a X)=a(m X)-X(a) m,
\end{aligned}
$$

Right $(A, L)$-module structures correspond to right $V L$-module structures and vice versa. For a right $(A, L)$-module $M$, we define Lie-Rinehart homology with coefficients in $M$ as

$$
H_{\bullet}(L, M):=\operatorname{Tor}_{\bullet}^{V L}(M, A) .
$$

Interestingly enough, every right $(A, L)$-module is an SaYD module with respect to the trivial coaction (cf. Remark 2.6):

Lemma 5.1. Let $M$ be a right $(A, L)$-module and define on $M$ the left $V L$-coaction $\Delta_{M}: M \rightarrow V L \otimes_{A} M, m \mapsto 1 \otimes_{A} m$. Then $M$ is a stable anti Yetter-Drinfel'd module.

Proof. Equipped with this coaction, $M$ is obviously stable, and also (17) is immediate (observe that left and right $A$-action on $M$ coincide). Hence it remains to show (18). With the left Hopf algebroid structure maps mentioned above, it is easy to see that on generators

$$
\Delta_{M}(m X)=1 \otimes_{A} m X=X_{-} X_{+(1)} \otimes_{A} m X_{+(2)}=X_{-} m_{(-1)} X_{+(1)} \otimes_{A} m_{(0)} X_{+(2)}
$$

holds for $X \in L$ and trivially on generators $a \in A$. For an element $u=a X_{1} \cdots X_{p}$, where $a \in A, X_{i} \in L$, one immediately obtains

$$
\begin{aligned}
\Delta(m u) & =1 \otimes_{A} m u^{\prime} X_{p} \\
& =\left(X_{p}\right)_{-}\left(X_{p}\right)_{+(1)} \otimes_{A} m u^{\prime}\left(X_{p}\right)_{+(2)} \\
& =\left(X_{p}\right)_{-}\left(m u^{\prime}\right)_{(-1)}\left(X_{p}\right)_{+(1)} \otimes_{A}\left(m u^{\prime}\right)_{(0)}\left(X_{p}\right)_{+(2)}
\end{aligned}
$$

for $u^{\prime}=a X_{1} \cdots X_{p-1}$. Using induction on $p$ and (9) one concludes that as desired we have $\Delta(m u)=u_{-} m_{(-1)} u_{+(1)} \otimes_{A} m_{(0)} u_{+(2)}$.

Recall that there is a canonical complex that computes $H_{\bullet}(L, M)$ whenever $L$ is $A$-projective. This is given by the exterior algebra $\wedge_{A}^{\bullet} L$ tensored over $A$ with $M$, 
with differential $\partial=\partial_{n}: M \otimes_{A} \bigwedge_{A}^{n} L \rightarrow M \otimes_{A} \bigwedge_{A}^{n-1} L$ defined by

$$
\begin{aligned}
\partial(m & \left.\otimes_{A} X_{1} \wedge \cdots \wedge X_{n}\right) \\
:= & \sum_{i=1}^{n}(-1)^{i+1} m X_{i} \otimes_{A} X_{1} \wedge \cdots \wedge \hat{X}_{i} \wedge \cdots \wedge X_{n} \\
& +\sum_{i<j}(-1)^{i+j} m \otimes_{A}\left[X_{i}, X_{j}\right] \wedge X_{1} \wedge \cdots \wedge \hat{X}_{i} \wedge \cdots \wedge \hat{X}_{j} \wedge \cdots \wedge X_{n} .
\end{aligned}
$$

The following theorem generalises [KoP, Thm. 3.13] to more general coefficients.

Theorem 5.2. Let $(A, L)$ be a Lie-Rinehart algebra, where $L$ is A-projective and $M$ a right $(A, L)$-module which is $A$-flat, seen also as a left $V L$-comodule as in Lemma 5.1. The map

$$
\Xi: m \otimes_{A} X_{1} \wedge \cdots \wedge X_{n} \mapsto \frac{1}{n !} \sum_{\sigma \in S_{n}}(-1)^{\sigma} X_{\sigma(1)} \otimes_{A} \cdots \otimes_{A} X_{\sigma(n)} \otimes_{A} m
$$

defines a morphism of mixed complexes

$$
\left(M \otimes_{A} \bigwedge_{A}^{\bullet} L, 0, \partial\right) \rightarrow\left(C^{\bullet}(V L, M), b, B\right)
$$

which induces natural isomorphisms

$$
\begin{aligned}
H^{\bullet}(V L, M) & \simeq M \otimes_{A} \bigwedge_{A}^{\bullet} L \\
H C^{\bullet}(V L, M) & \simeq \operatorname{ker} \partial_{\bullet} \oplus H_{\bullet-2}(L, M) \oplus H_{\bullet-4}(L, M) \oplus \cdots
\end{aligned}
$$

Proof. The first part of the theorem and the first isomorphism follow immediately by the form of the cosimplicial operators in (24) for a trivial coaction, combined with the analogous result for $M=A$ from $[\mathbf{K o P}]$ and the flatness assumption on $M$.

To prove the second isomorphism, we need to show that $\Xi$ intertwines the horizontal differential $B$ with $\partial$. This will be done by explicitly applying the coinvariants functor and the results in Section 3. Let $\tilde{B}: B^{\bullet}(V L, M) \rightarrow B^{\bullet-1}(V L, M)$ denote the horizontal differentials of the mixed complex associated to the cocyclic module from Lemma 3.1. Hence $\tilde{B}=N \sigma_{-1}(1-\lambda)$, where $\lambda:=(-1)^{n} \tau_{n}, N:=\sum_{i=0}^{n} \lambda^{i}$, and $\sigma_{-1}:=\sigma_{n-1} \tau_{n}$. Explicitly, we obtain

$$
\begin{aligned}
& \tilde{B}\left(u_{0} \otimes_{A} \cdots \otimes_{A} u_{n} \otimes_{A} m\right) \\
& =\sum_{i=0}^{n}\left((-1)^{n i} \epsilon\left(u_{0}\right) u_{i+1} \otimes_{A} \cdots \otimes_{A} u_{n} \otimes_{A} u_{1} \otimes_{A} \cdots \otimes_{A} u_{i-1} \otimes_{A} m\right. \\
& \left.\quad-(-1)^{n(i-1)} \epsilon\left(u_{n}\right) u_{i+1} \otimes_{A} \cdots \otimes_{A} u_{n-1} \otimes_{A} u_{0} \otimes_{A} \cdots \otimes_{A} u_{i-1} \otimes_{A} m\right) .
\end{aligned}
$$

Note that $B^{n}(V L, M) \cong C^{n+1}(V L, M)$ as $(A, A)$-bimodules in this example. From our general considerations in Section 3, we have $B \circ \phi \circ \pi=\phi \circ \pi \circ \tilde{B}$, where $\pi$ is the canonical projection $B^{\bullet}(U, M) \rightarrow U^{\otimes_{A} \bullet+1} \otimes_{U^{\text {op }}} M$ and $\phi: U^{\otimes_{A} \bullet+1} \otimes_{U^{\text {op }}} M \rightarrow$ $C^{n}(V L, M)$ is the isomorphism (21). Using its right inverse mentioned in the proof of Proposition 3.4, it is seen that

$$
\Xi\left(m \otimes_{A} X_{1} \wedge \cdots \wedge X_{n}\right)=\phi\left(\pi\left(\frac{1}{n !} \sum_{\sigma \in S_{n}}(-1)^{\sigma} 1 \otimes_{A} X_{\sigma(1)} \otimes_{A} \cdots \otimes_{A} X_{\sigma(n)} \otimes_{A} m\right)\right) .
$$


Hence, because $L \subset \operatorname{ker} \epsilon$ we can compute

$$
\begin{aligned}
& B(\left.\Xi\left(m \otimes_{A} X_{1} \wedge \cdots \wedge X_{n}\right)\right) \\
&= \phi\left(\pi\left(\tilde{B}\left(\frac{1}{n !} \sum_{\sigma \in S_{n}}(-1)^{\sigma} 1 \otimes_{A} X_{\sigma(1)} \otimes_{A} \cdots \otimes_{A} X_{\sigma(n)} \otimes_{A} m\right)\right)\right) \\
&= \phi\left(\pi\left(\frac{1}{(n-1) !} \sum_{\sigma \in S_{n}}(-1)^{\sigma} X_{\sigma(1)} \otimes_{A} \cdots \otimes_{A} X_{\sigma(n)} \otimes_{A} m\right)\right) \\
&= \frac{1}{(n-1) !} \sum_{\sigma \in S_{n}}(-1)^{\sigma} X_{\sigma(1)} \cdot\left(X_{\sigma(2)} \otimes_{A} \cdots \otimes_{A} X_{\sigma(n)}\right) \otimes_{A} m X_{\sigma(1)+}, \\
&= \frac{1}{(n-1) !} \sum_{\sigma \in S_{n}}(-1)^{\sigma} X_{\sigma(2)} \otimes_{A} \cdots \otimes_{A} X_{\sigma(n)} \otimes_{A} m X_{\sigma(1)} \\
&-\frac{1}{(n-1) !} \sum_{i=1}^{n} \sum_{\sigma \in S_{n}}(-1)^{\sigma} X_{\sigma(2)} \otimes_{A} \cdots \otimes_{A} X_{\sigma(1)} X_{\sigma(i)} \otimes_{A} \cdots \otimes_{A} X_{\sigma(n)} \otimes_{A} m \\
&= \Xi\left(\sum_{i=1}^{n}(-1)^{i+1} m X_{i} \otimes_{A} X_{1} \wedge \cdots \wedge \hat{X}_{i} \wedge \cdots \wedge X_{n}\right. \\
&\left.\quad \quad \quad+\sum_{i<j}(-1)^{i+j} m \otimes_{A}\left[X_{i}, X_{j}\right] \wedge X_{1} \wedge \cdots \wedge \hat{X}_{i} \wedge \cdots \wedge \hat{X}_{j} \wedge \cdots \wedge X_{n}\right) \\
&=\Xi\left(\partial\left(m \otimes_{A} X_{1} \wedge \cdots \wedge X_{n}\right)\right),
\end{aligned}
$$

where denotes the diagonal action via the coproduct. This completes the proof.

Remark 5.3. Note that combining the preceding theorem with Proposition 4.3 as well as (28) relates the Hopf-cyclic cohomology of $V L$ with the Hopf algebroid homology, that is, the simplicial theory of the dual Hopf-cyclic homology:

$$
\begin{aligned}
H C \cdot(V L, M) & \simeq \operatorname{ker} \partial \bullet \oplus H_{\bullet-2}(L, M) \oplus H_{\bullet-4}(L, M) \oplus \cdots \\
& \simeq \operatorname{ker} \partial \bullet \oplus \operatorname{Tor}_{\bullet-2}(M, A) \oplus \operatorname{Tor}_{\bullet-4} V L \\
& \simeq \operatorname{ker} \partial \bullet \oplus H_{\bullet-2}(V L, M) \oplus H_{\bullet-4}(V L, M) \oplus \cdots
\end{aligned}
$$

\subsection{Twisted cyclic homology}

Recall from [Sch2] that $U=A^{\mathrm{e}}$ is for any $k$-algebra $A$ a left Hopf algebroid over $A$ with structure maps

$$
s(a):=a \otimes_{k} 1, t(b):=1 \otimes_{k} b, \Delta\left(a \otimes_{k} b\right):=\left(a \otimes_{k} 1\right) \otimes_{A}\left(1 \otimes_{k} b\right), \epsilon\left(a \otimes_{k} b\right):=a b .
$$

The inverse of the Hopf-Galois map is given by

$$
\left(a \otimes_{k} b\right)_{+} \otimes_{A^{\mathrm{op}}}\left(a \otimes_{k} b\right)_{-}:=\left(a \otimes_{k} 1\right) \otimes_{A^{\mathrm{op}}}\left(b \otimes_{k} 1\right) .
$$

Any algebra endomorphism $\sigma: A \rightarrow A$ defines a right $A^{\mathrm{e}}$-module $A_{\sigma}$ which is $A$ as a $k$-module with the right action

$$
x\left(a \otimes_{k} b\right):=b x \sigma(a), \quad a, x \in A, b \in A^{\mathrm{op}} .
$$

Furthermore, define a left $A^{\mathrm{e}}$-comodule structure on $A_{\sigma}$ by

$$
A_{\sigma} \rightarrow A^{\mathrm{e}} \otimes_{A} A_{\sigma}, \quad x \mapsto\left(x \otimes_{k} 1\right) \otimes_{A} 1,
$$

which reduces to the map $A_{\sigma} \rightarrow A^{\mathrm{e}}, x \mapsto x \otimes_{k} 1$. With this $A^{\mathrm{e}}$-action and $A^{\mathrm{e}}$-coaction on $A_{\sigma}$, we have $b x=b \bullet x$, but $x a$ is different from $x \triangleleft a$ unless $\sigma=\operatorname{id}_{A}$. So if $\sigma$ is not the identity, the condition (17) is not fulfilled and $A_{\sigma}$ is therefore not an anti Yetter-Drinfel'd module. 
Under the isomorphism $C .\left(A^{\mathrm{e}}, A_{\sigma}\right)=A_{\sigma} \otimes_{A^{\mathrm{op}}} A^{\mathrm{e} \otimes_{A^{\mathrm{op}} n}} \simeq A_{\sigma} \otimes_{k} A^{\otimes_{k} n}$ given by

$$
x \otimes_{A^{\mathrm{op}}}\left(a_{1} \otimes_{k} b_{1}\right) \otimes_{A^{\mathrm{op}}} \cdots \otimes_{A^{\mathrm{op}}}\left(a_{n} \otimes_{k} b_{n}\right) \mapsto b_{n} \cdots b_{1} x \otimes_{k} a_{1} \otimes_{k} \cdots \otimes_{k} a_{n},
$$

the para-cyclic operators (26) become

$$
\begin{aligned}
& d_{i}\left(x \otimes_{k} y\right)= \begin{cases}a_{n} x \otimes_{k} a_{1} \otimes_{k} \cdots \otimes_{k} a_{n-1} & \text { if } i=0, \\
x \otimes_{k} \cdots \otimes_{k} a_{n-i} a_{n-i+1} \otimes_{k} \cdots & \text { if } 1 \leqslant i \leqslant n-1, \\
x \sigma\left(a_{1}\right) \otimes_{k} a_{2} \otimes_{k} \cdots \otimes_{k} a_{n} & \text { if } i=n,\end{cases} \\
& s_{i}\left(x \otimes_{k} y\right)= \begin{cases}x \otimes_{k} a_{1} \otimes_{k} \cdots \otimes_{k} a_{n} \otimes_{k} 1 & \text { if } i=0, \\
x \otimes_{k} \cdots \otimes_{k} a_{n-i} \otimes_{k} 1 \otimes_{k} a_{n-i+1} \otimes_{k} & \text { if } 1 \leqslant i \leqslant n-1, \\
x \otimes_{k} 1 \otimes_{k} a_{1} \otimes_{k} \cdots \otimes_{k} a_{n} & \text { if } i=n,\end{cases} \\
& t_{n}\left(x \otimes_{k} y\right)=\sigma\left(a_{1}\right) \otimes_{k} a_{2} \otimes_{k} \cdots \otimes_{k} a_{n} \otimes_{k} x,
\end{aligned}
$$

where we abbreviate $y:=a_{1} \otimes_{k} \cdots \otimes_{k} a_{n}$. In particular, one has

$$
t_{n}^{n+1}=\sigma \otimes_{k} \cdots \otimes_{k} \sigma
$$

so $C_{\bullet}\left(A^{\mathrm{e}}, A_{\sigma}\right)$ is cyclic if and only if $\sigma=\mathrm{id}$ (in which case $A_{\sigma}$ is an SaYD module).

However, there are situations in which the canonical projection from $C_{\bullet}\left(A^{\mathrm{e}}, A_{\sigma}\right)$ onto its associated cyclic $k$-module $C \cdot\left(A^{\mathrm{e}}, A_{\sigma}\right) / \mathrm{im}\left(\mathrm{id}-t_{\bullet^{\bullet+1}}\right)$ is a quasi-isomorphism of the underlying simplicial $k$-modules; see, e.g., [HaKr, Prop. 2.1], which implies:

Theorem 5.4. If $k$ is a field and $\sigma$ is a diagonalisable automorphism of $A$, then

$$
H_{\bullet}\left(A^{\mathrm{e}}, A_{\sigma}\right) \simeq H_{\bullet}\left(A, A_{\sigma}\right) .
$$

Here the right-hand side denotes the Hochschild homology of $A$ with coefficients in the $(A, A)$-bimodule $A_{\sigma}$. The resulting cyclic homology $H C_{\bullet}^{\sigma}(A):=H C \cdot\left(A^{\mathrm{e}}, A_{\sigma}\right)$ has been first considered in $[\mathbf{K u M u T u}]$ under the name $\sigma$-twisted cyclic homology and has served as yet another guiding example of generalised cyclic homology theories. It can be also expressed as the Hopf-cyclic homology of the $k \mathbb{Z}$-module algebra $A$ (where $k \mathbb{Z}$ acts via $\sigma$ ), but the above presentation seems more natural and stresses the way it originates as a deformation of $H C$. $(A)$. We therefore consider it an important example that motivates both the generalisation of Hopf-cyclic (co)homology from Hopf algebras to Hopf algebroids and the necessity to consider coefficients beyond SaYD modules. The above shows how to extend the construction of [KuMuTu] to arbitrary $(A, A)$-bimodules assuming the existence of an $A^{\mathrm{e}}$-coaction on the coefficients.

\section{Acknowledgements}

It is a pleasure to thank Tomasz Brzeziński, Atabey Kaygun, and Jean-Louis Loday for helpful comments. Niels Kowalzig is supported by an I.H.É.S. visiting grant. Ulrich Krähmer is supported by the EPSRC fellowship EP/E/043267/1 and partially by the Marie Curie PIRSES-GA-2008-230836 network and the Polish Government Grant N201 177033. 


\section{References}

[B1] G. Böhm, Galois theory for Hopf algebroids, Ann. Univ. Ferrara Sez. VII (N.S.) 51 (2005), no. 1, 233-262.

[B2] G. Böhm, Hopf algebroids, Handbook of algebra, Vol. 6, North-Holland, Amsterdam, 2009, pp. 173-235.

[BŞ1] G. Böhm and D. Ştefan, A categorical approach to cyclic duality, preprint (2009), arXiv:0910.4622, to appear in J. Noncommut. Geom.

[BŞ2] G. Böhm and D. Ştefan, (Co)cyclic (co)homology of bialgebroids: an approach via (co)monads, Comm. Math. Phys. 282 (2008), no. 1, 239286.

[BSz] G. Böhm and K. Szlachányi, Hopf algebroids with bijective antipodes: axioms, integrals, and duals, J. Algebra 274 (2004), no. 2, 708-750.

[BrzWi] T. Brzeziński and R. Wisbauer, Corings and comodules, London Math. Soc. Lect. Note Series 309, Cambridge Univ. Press, Cambridge, 2003.

[C] A. Connes, Cohomologie cyclique et foncteurs Ext ${ }^{n}$, C. R. Acad. Sci. Paris Sér. I Math. 296 (1983), no. 23, 953-958.

[CM1] A. Connes and H. Moscovici, Differentiable cyclic cohomology and Hopf algebraic structures in transverse geometry, Essays on geometry and related topics, Vol. 1, 2, Monogr. Enseign. Math. 38, Enseignement Math., Geneva, 2001, pp. 217-255.

[CM2] A. Connes and H. Moscovici, Hopf algebras, cyclic cohomology and the transverse index theorem, Comm. Math. Phys. 198 (1998), no. 1, 199-246.

[Cr] M. Crainic, Cyclic cohomology of Hopf algebras, J. Pure Appl. Algebra 166 (2002), no. 1-2, 29-66.

[E] A. Elmendorf, A simple formula for cyclic duality, Proc. Amer. Math. Soc. 118 (1993), no. 3, 709-711.

[FTs] B. Feĭgin and B. Tsygan, Additive $K$-theory, $K$-theory, arithmetic and geometry (Moscow, 1984-1986), Lecture Notes in Math. 1289, Springer-Verlag, New York, 1987, pp. 67-209.

[FiL] Z. Fiedorowicz and J.-L. Loday, Crossed simplicial groups and their associated homology, Trans. Amer. Math. Soc. 326 (1991), no. 1, 5787.

[HKhRS1] P. Hajac, M. Khalkhali, B. Rangipour and Y. Sommerhäuser, Stable anti-Yetter-Drinfeld modules, C. R. Math. Acad. Sci. Paris 338 (2004), no. 8, 587-590.

[HKhRS2] P. Hajac, M. Khalkhali, B. Rangipour and Y. Sommerhäuser, Hopfcyclic homology and cohomology with coefficients, C. R. Math. Acad. Sci. Paris 338 (2004), no. 9, 667-672.

[HaKr] T. Hadfield and U. Krähmer, Twisted homology of quantum $S L(2)$, K-Theory 34 (2005), no. 4, 327-360.

[HasR] M. Hassanzadeh and B. Rangipour, Equivariant Hopf Galois extensions and Hopf cyclic cohomology, preprint (2010), arXiv:1010.5818. 
[Hue] J. Huebschmann, Duality for Lie-Rinehart algebras and the modular class, J. Reine Angew. Math. 510 (1999), 103-159.

[KSz] L. Kadison and K. Szlachányi, Bialgebroid actions on depth two extensions and duality, Adv. Math. 179 (2003), no. 1, 75-121.

[Ka1] A. Kaygun, Bialgebra cyclic homology with coefficients, K-Theory 34 (2005), no. 2, 151-194.

[Ka2] A. Kaygun, The universal Hopf-cyclic theory, J. Noncommut. Geom. 2 (2008), no. 3, 333-351.

[KhR] M. Khalkhali and B. Rangipour, Para-Hopf algebroids and their cyclic cohomology, Lett. Math. Phys. 70 (2004), no. 3, 259-272.

[Ko] N. Kowalzig, Hopf algebroids and their cyclic theory, Ph.D. thesis, Universiteit Utrecht, 2009.

[KoKr] N. Kowalzig and U. Krähmer, Duality and products in algebraic (co)homology theories, J. Algebra 323 (2010), no. 7, 2063-2081.

[KoP] N. Kowalzig and H. Posthuma, The cyclic theory of Hopf algebroids, preprint (2009), arXiv:0904.4736, to appear in J. Noncommutat. Geom.

[KuMuTu] J. Kustermans, G. Murphy and L. Tuset, Differential calculi over quantum groups and twisted cyclic cocycles, J. Geom. Phys. 44 (2003), no. $4,570-594$.

[L] J.-L. Loday, Cyclic homology, second ed., Grundlehren der Mathematischen Wissenschaften 301, Springer-Verlag, New York, 1998.

[Ma] T. Maszczyk, Noncommutative geometry through monoidal categories, preprint (2006), arXiv:math/0611806.

[Ri] G. Rinehart, Differential forms on general commutative algebras, Trans. Amer. Math. Soc. 108 (1963), 195-222.

[Sch1] P. Schauenburg, Bialgebras over noncommutative rings and a structure theorem for Hopf bimodules, Appl. Categ. Structures 6 (1998), no. 2, 193-222.

[Sch2] P. Schauenburg, Duals and doubles of quantum groupoids $\left(\times_{R}\right.$-Hopf algebras), in New trends in Hopf algebra theory (La Falda, 1999), Contemp. Math. 267, Amer. Math. Soc., Providence, RI, 2000, pp. 273-299.

[T] M. Takeuchi, Groups of algebras over $A \otimes \bar{A}$, J. Math. Soc. Japan 29 (1977), no. 3, 459-492.

Niels Kowalzig kowalzig@ihes.fr

Institut des Hautes Études Scientifiques, Le Bois-Marie, 35, route de Chartres, 91440 Bures-sur-Yvette, France

Ulrich Krähmer ulrich.kraehmer@glasgow.ac.uk

University of Glasgow, School of Mathematics and Statistics, University Gardens, Glasgow G12 8QW, Scotland 